\title{
RBF fuzzy reasoning flexible decision making method oriented to statistical information
}

\author{
Tao Duan, Jing Huang \\ 1.Henan University of Chinese Medicine, ZhengZhou, China \\ 59203896@qq.com
}

Key words: statistical information; hierarchical method; fuzzy reasoning; decision making

\begin{abstract}
Integrated fuzzy logarithm preference programming proposed by the paper can be used to treat AHP and ANP problem effectively and reliably. The method improves FPP defects, such as comparative matrix is treated one by one and weight generated by upper triangular judgment of matrix is different from that by lower triangular judgment. In the five examples, the result shows because of extra constraint, FPP method makes upper triangular judgment and lower triangular judgment obtain priority and ordering discrepancy. Because upper triangular judgment provides the same information as lower triangular judgment, priority will also be the same. The proposed method of using multiplicative constraints can obtain the same priority and ordering; moreover, through multi-objective programming, the proposed method can solve all comparative matrixes at the same time while their complexity is not related to the number of comparative matrixes.
\end{abstract}

\section{Introduction}

Analytic Hierarchy Process (AHP) [1] has been widely applied to multi-criteria decision making to measure visible and invisible criteria; AHP aims to decompose decision-making process into hierarchical structure and supposes that different hierarchical criteria are independent from each other. However, when the method is used to judge interval, inconsistent problem will appear, making it difficult to generate weight value [2]. Literature [3] proposes Lexicographic Orderings Goal Programming (LOGP), using deviation variables in AHP interval to solve inconsistent problem. Literature [4] proposes Fuzzy Preference Programming (FPP), obtaining clear priority from intervals or fuzzy comparative matrix by means of introducing tolerance parameter, but because weight is generated by extra constraints in FPP, different priorities and orderings [5] will be obtained from upper and lower triangular judgments.

\section{Fuzzy preference programming model}

FPP is used to treat fuzzy comparative matrix, especially those inconsistent fuzzy matrixes. Assumed that $\tilde{A}=\left\{a_{i j}\right\}$ represents pairing fuzzy comparative matrix with $n_{\text {criteria. }}$

$$
\tilde{A}=\left[\begin{array}{ccccc}
1 & \tilde{a}_{12} & \tilde{a}_{13} & \cdots & \tilde{a}_{1 n} \\
\tilde{a}_{21} & 1 & \tilde{a}_{23} & \cdots & \tilde{a}_{2 n} \\
\tilde{a}_{31} & \tilde{a}_{32} & 1 & \cdots & \tilde{a}_{3 n} \\
\vdots & \vdots & \vdots & \vdots & \vdots \\
\tilde{a}_{n 1} & \tilde{a}_{n 2} & \tilde{a}_{n 3} & \cdots & 1
\end{array}\right]
$$

Where, $\tilde{a}_{i j}=\left(l_{i j}, m_{i j}, u_{i j}\right), \quad \tilde{a}_{j i}=1 / \tilde{a}_{i j}=\left(1 / u_{i j}, 1 / m_{i j}, 1 / l_{i j}\right), l_{i j}, m_{i j}$ and $u_{i j}$ are low, medium and high borders of uncertain judgments corresponded to $i=1,2, \ldots, n-1, j=2,3, \ldots, n, j>i$.

When interval judgments are consistent, prioritized vector $w=\left(w_{1}, w_{2}, \ldots, w_{n}\right)^{T}$, its elements 
satisfy the inequation:

$$
l_{i j}(\alpha) \leq \frac{w_{i}}{w_{j}} \leq u_{i j}(\alpha), i=1,2, \ldots, n-1, j=2,3, \ldots, n, j>i
$$

Where, $\alpha$-cut range is $0 \leq \alpha \leq 1$.

$$
\begin{aligned}
& l_{i j}(\alpha)=l_{i j}+\alpha\left(m_{i j}-l_{i j}\right) \\
& u_{i j}(\alpha)=u_{i j}+\alpha\left(m_{i j}-u_{i j}\right)
\end{aligned}
$$

When judgments are inconsistent, a prioritized matrix satisfying all fuzzy judgments can be represented approximately as follows:

$$
l_{i j}(\alpha) \tilde{\leq} \frac{w_{i}}{w_{j}} \tilde{\leq} u_{i j}(\alpha), i=1,2, \ldots, n-1, j=2,3, \ldots, n, j>i
$$

Where, $\tilde{\leq}$ means “fuzzy or equal”. Inequation (4) can be represented by a group of unilateral fuzzy constraints:

$$
\left\{\begin{array}{c}
w_{i}-w_{j} u_{i j}(\alpha) \tilde{\leq} 0 \\
-w_{i}+w_{j} l_{i j}(\alpha) \tilde{\leq} 0
\end{array}\right.
$$

Aforementioned $n(n-1)$ fuzzy constraint sets shall be provided by the following matrix form:

$$
R W \tilde{\leq} 0
$$

Where, matrix

$$
R \in \mathfrak{R}^{m \times n}, m=n(n-1)
$$

To measure the satisfaction of consistent fuzzy judgment, liner member function shall be adopted as follows:

$$
\mu_{k}\left(R_{k} w\right)= \begin{cases}1-\frac{R_{k} w}{d_{k}} & R_{k} w \leq d_{k} \\ 0 & R_{k} w \geq d_{k}\end{cases}
$$

Where, $d_{k}$ is the K-th constrained tolerance parameter, and provided by $k=1,2, \ldots, m$ decision-making process. Generally, $d_{k}$ is set as 1 .

FPP method is to select a prioritized vector and the vector member has the highest level, which is described as follows:

$$
\lambda=\max _{w}\left[\min \left\{\mu_{1}\left(R_{1} w\right), \mu_{2}\left(R_{2} w\right), \ldots, \mu_{m}\left(R_{n} w\right)\right\} \mid \sum_{i=1}^{n} w_{i}=1\right]
$$

Every priority problem of $\alpha$-cut level can be solved by solving the following liner programming problem: Max $\lambda$, where,

$$
\begin{aligned}
& d_{k} \lambda+w_{i}-u_{i j}(\alpha) w_{j} \leq d_{k} \\
& d_{k} \lambda-w_{i}+l_{i j}(\alpha) w_{j} \leq d_{k} \\
& \sum_{i=1}^{n} w_{i}=1, w_{i}>0
\end{aligned}
$$

When $\lambda$ is equal to 1 , the comparative matrix is consistent; but when $\lambda$ is less than 1 , comparative matrix is inconsistent.

FPP uses $d_{k}$, the tolerance parameter in inequation (10) and (11), to determine and treat inconsistency, but, because of extra constraint in inequation (12), FPP has the defects of obtaining 
two different weights and orderings respectively from the upper triangular and lower triangular judgments of pairing comparative matrixes in fuzzy AHP. Because extra constraint of FPP, fuzzy ANP also has the defects of obtaining different weights from judgment of upper triangular and lower triangular. Therefore, the paper provides an integrated fuzzy logarithm preference programming with multiplicative constraints for AHP and ANP, adopting multi-objective programming to obtain efficient solution at the same time without considering the number of comparative matrixes.

\section{Method proposal}

The proposed method can be used to obtain reasonable weight and same priority ranking through selecting logarithm of interval judgment and treating upper triangular and lower triangular judgments of comparative matrixes in interval. Using multiplicative constraint to take the place of extra constraint, it can be got such as $v=\left(v_{1}, \ldots, v_{n}\right)^{T}$ weight vector, multiplication type attribute is $\prod_{i=1}^{n} v_{i}=1$ and arithmetic equation is $\sum_{i=1}^{n} \ln v_{i}=0$. By means of the strategy, different weight problem (problem of different priority rankings obtained from the upper and lower triangular judgments of the same matrix) can be treated well by using these multiplicative constraints.

Supposed that there are s pairs of comparative matrixes in AHP and ANP problems, to get all priority sets of s pairs of comparative matrixes at the same time, following s number of objectives are required to be maximized.

$$
\begin{aligned}
& \operatorname{Max}_{1} \\
& \operatorname{Max} \lambda_{2} \\
& \cdot \\
& \text { - } \\
& \text { Max } \lambda_{s}
\end{aligned}
$$

Where,

$$
\begin{aligned}
& d_{p k} \lambda_{p}+x_{p i}-x_{p j}-\ln u_{p i j}(\alpha) \leq d_{p k} \\
& d_{p k} \lambda_{p}-x_{p i}+x_{p j}+\ln l_{p i j}(\alpha) \leq d_{p k} \\
& x_{p 1}=0
\end{aligned}
$$

$$
p=1,2, \ldots, s, \quad k=1,2, \ldots, m_{p}, \quad i=1,2, \ldots, n-1, \quad j=i+1, i+2, \ldots, n, \quad m_{p}=n_{p}\left(n_{p}-1\right), \quad x_{p j} \text { is }
$$
unconstrained, $\lambda_{p}$ is the satisfaction of the P-th pair of comparative matrixes, $d_{p k}$ is the k-th constrained tolerance parameter of the p-th pair of comparative matrixes.

The above multi-objective problem can be rewritten as single objective problem by double-stage multi-objective programming, to guarantee the non-dominated and balanced solution. Thus, it can help to generate better balance result for every objective. Member function of each objective can be defined as follows:

$$
\mu_{p}\left(\lambda_{p}\right)=\frac{\lambda_{p}-\lambda_{p}{ }^{-}}{\lambda_{p}{ }^{+}-\lambda_{p}{ }^{-}}=\lambda_{p}, p=1,2, \ldots, s
$$

Where, $\lambda_{p}{ }^{+}$, the ideal solution of the $\mathrm{p}$-th objective, and $\lambda_{p}{ }^{-}$, the anti-ideal solution are 1 and 0 respectively; at the first stage, non-compensatory "minimum" operator is used, namely $\lambda^{(1)}=\min \mu_{p}\left(\lambda_{p}\right)$, merges multi-objective programming problem as single-objective programming problem.

Following are the respective introductions to the two stages of upper and lower triangular 
judgment models, which is similar in treatment process.

\section{Example analysis}

The part will conduct example analysis on proposed algorithm, including interval AHP, interval ANP, fuzzy AHP and fuzzy ANP, which can overcome the defects that FPP generates different weights from lower and upper triangular matrixes, moreover, weights generated from the whole matrix of each example are also provided. For simplicity, assume $d_{p k}$ in fuzzy AHP and ANP as 1, $\alpha$ as 0.

Interval AHP is as shown in Fig. 1, someone wants to use one of the four investment schemes to invest: bank deposit (BD), bond (DB), government bond (GB) or share (SH). In the four investment schemes, he can only select one based on the four criteria: return (RE), risk (RI), income tax benefit (TB) and liquidity ( $\mathrm{Li}$ ), this way, there will be 5 comparative matrixes for all criteria, which is shown as Table 1-Table 5.

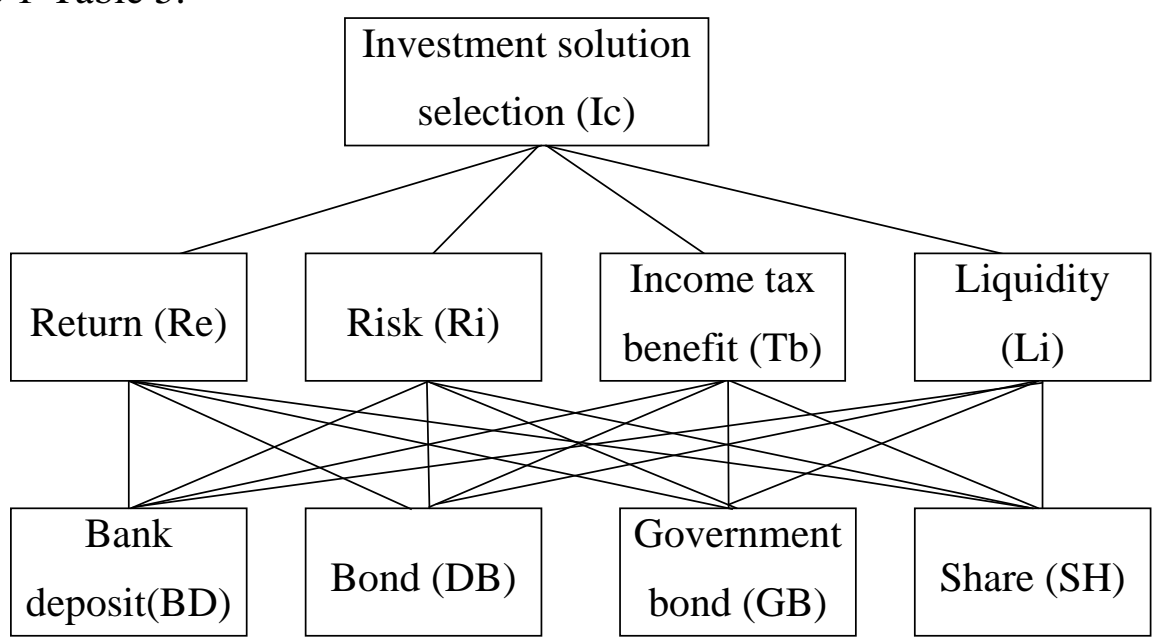

Fig. 1 Decision-making Level in Investment Aspect

Table 1 Comparative Matrixes in Interval of All Ic Criteria

\begin{tabular}{|c|c|c|c|c|}
\hline $\mathrm{Ic}$ & $\mathrm{Re}$ & $\mathrm{Ri}$ & $\mathrm{Tb}$ & $\mathrm{Li}$ \\
\hline $\mathrm{Re}$ & 1 & {$[3,4]$} & {$[5,6]$} & {$[6,7]$} \\
\hline $\mathrm{Ri}$ & {$[1 / 4,1 / 3]$} & 1 & {$[4,5]$} & {$[5,6]$} \\
\hline $\mathrm{Tb}$ & {$[1 / 6,1 / 5]$} & {$[1 / 5,1 / 4]$} & 1 & {$[3,4]$} \\
\hline $\mathrm{Li}$ & {$[1 / 7,1 / 6]$} & {$[1 / 6,1 / 5]$} & {$[1 / 4,1 / 3]$} & 1 \\
\hline
\end{tabular}

Table 2 Interval Comparative Matrixes of All Re Criteria

\begin{tabular}{|c|c|c|c|c|}
\hline Re & BD & DB & GB & SH \\
\hline BD & 1 & {$[1 / 4,1 / 3]$} & {$[3,4]$} & {$[1 / 6,1 / 5]$} \\
\hline DB & {$[3,4]$} & 1 & {$[6,7]$} & {$[1 / 5,1 / 4]$} \\
\hline GB & {$[1 / 4,1 / 3]$} & {$[1 / 7,1 / 6]$} & 1 & {$[1 / 7,1 / 6]$} \\
\hline SH & {$[5,6]$} & {$[4,5]$} & {$[6,7]$} & 1 \\
\hline
\end{tabular}

Table 3 Comparative Matrixes in Interval of All Ri Criteria

\begin{tabular}{|c|c|c|c|c|}
\hline Ri & BD & DB & GB & SH \\
\hline BD & 1 & {$[3,4]$} & {$[4,5]$} & {$[6,7]$} \\
\hline DB & {$[1 / 4,1 / 3]$} & 1 & {$[3,4]$} & {$[5,6]$} \\
\hline GB & {$[1 / 5,1 / 4]$} & {$[1 / 4,1 / 3]$} & 1 & {$[4,5]$} \\
\hline SH & {$[1 / 7,1 / 6]$} & {$[1 / 6,1 / 5]$} & {$[1 / 5,1 / 4]$} & 1 \\
\hline
\end{tabular}


Table 4 Comparative Matrixes in Interval of All Tb Criteria

\begin{tabular}{|c|c|c|c|c|}
\hline Tb & BD & DB & GB & SH \\
\hline BD & 1 & 1 & {$[1 / 6,1 / 5]$} & {$[1 / 4,1 / 3]$} \\
\hline DB & 1 & 1 & {$[1 / 6,1 / 5]$} & {$[1 / 4,1 / 3]$} \\
\hline GB & {$[5,6]$} & {$[5,6]$} & 1 & {$[4,5]$} \\
\hline SH & {$[3,4]$} & {$[3,4]$} & {$[1 / 5,1 / 4]$} & 1 \\
\hline
\end{tabular}

Table 5 Comparative Matrixes in Interval of All Li Criteria

\begin{tabular}{|c|c|c|c|c|}
\hline Li & BD & DB & GB & SH \\
\hline BD & 1 & {$[3,4]$} & 6 & {$[6,7]$} \\
\hline DB & {$[1 / 4,1 / 3]$} & 1 & {$[3,4]$} & {$[3,4]$} \\
\hline GB & {$[1 / 6,1 / 5]$} & {$[1 / 4,1 / 3]$} & 1 & {$[3,4]$} \\
\hline SH & {$[1 / 7,1 / 6]$} & {$[1 / 4,1 / 3]$} & {$[1 / 4,1 / 3]$} & 1 \\
\hline
\end{tabular}

FPP treats each matrix one by one, and the proposed method can solve all matrixes at the same time, with generated comparative weights shown in Table 6. Using FPP, the weight vector of upper triangular matrix is: $w=(0.257,0.204,0.122,0.417)^{T}$, priority ordering is: $S H>B D>D B>G B$, the weight vector of lower triangular matrix is: $w=(0.241,0.185,0.157,0.417)^{T}$, priority ordering is: $S H>B D>D B>G B$, obviously, the prioritization matrixes obtained from upper triangular matrix and lower triangular matrix or the whole matrix are all quite different.

Table 6 Weight Comparison of Interval AHP

\begin{tabular}{|c|c|c|c|c|c|c|c|c|}
\hline \multirow{2}{*}{ Matrix } & \multicolumn{4}{|c|}{ Proposed method } & \multicolumn{4}{c|}{ FPP } \\
\cline { 2 - 9 } & BD & DB & GB & SH & BD & DB & GB & SH \\
\hline Upper triangular & 0.254 & 0.226 & 0.144 & 0.376 & 0.257 & 0.204 & 0.122 & 0.417 \\
\hline Lower triangular & 0.254 & 0.226 & 0.144 & 0.376 & 0.241 & 0.185 & 0.157 & 0.417 \\
\hline Whole matrix & 0.254 & 0.226 & 0.144 & 0.376 & 0.263 & 0.199 & 0.131 & 0.407 \\
\hline
\end{tabular}

Seen from Table 6, using the proposed method can obtain the same weight and ordering from upper and lower triangular judgments of matrixes, and the weight vector is: $w=(0.254,0.226,0.144,0.376)^{T}$, the ordering is: $S H>B D>D B>G B$.

\section{Conclusion}

The research hopes the proposed method can be expanded to other AHP or ANP method with extra constraints, it also hopes the method can be applied to more examples. Based on the aforementioned analysis, double-stage fuzzy logarithm preference programming method of fuzzy AHP optimum network analysis is proposed. Since all priorities are generated at the same time, the proposed method can be used to treat complex structure problems while it is not related to the number of comparative matrixes; depending on multiplicative constraints [14] to take the place of extra constraints, obtaining the same priority from upper and lower triangular judgments, numerical examples indicates the effectiveness and reliability of the proposed method.

\section{Reference}

[1] Kong F, Liu H. Fuzzy RBF neural network model for multiple attribute decision making[C]// International Conference on Neural Information Processing. Springer-Verlag, 2006:1046-1054.

[2] Alam J, Hassan M, Khan A, et al. Robust fuzzy RBF network based image segmentation and intelligent decision making system for carotid artery ultrasound images[J]. Neurocomputing, 2015, 151(11):745-755.

[3] Montibeller G, Ensslin L. Reasoning maps for decision aid: an integrated approach for problem-structuring and multi-criteria evaluation[J]. Journal of the Operational Research Society, 2008, 59(5):575-589.

[4] Ebrahimnejad S, Mousavi S M, Tavakkoli-Moghaddam R, et al. A novel two-phase group 
decision making approach for construction project selection in a fuzzy environment[J]. Applied Mathematical Modelling, 2012, 36(9):4197-4217.

[5] Shen C Y, Yu K T. Enhancing the efficacy of supplier selection decision-making on the initial stage of new product development: A hybrid fuzzy approach considering the strategic and operational factors simultaneously[J]. Expert Systems with Applications, 2009, 36(8):11271-11281. 